\title{
Genetic analysis of Verticillium wilt resistance in a backcross inbred line population and a meta-analysis of quantitative trait loci for disease resistance in cotton
}

\author{
Jinfa Zhang ${ }^{1 *}$, Jiwen $\mathrm{Yu}^{2^{*}}$, Wenfeng $\mathrm{Pei}^{2}$, Xingli $\mathrm{Li}^{2}$, Joseph Said ${ }^{1}$, Mingzhou Song ${ }^{3}$ and Soum Sanogo ${ }^{4}$
}

\begin{abstract}
Background: Verticillium wilt (VW) and Fusarium wilt (FW), caused by the soil-borne fungi Verticillium dahliae and Fusarium oxysporum f. sp. vasinfectum, respectively, are two most destructive diseases in cotton production worldwide. Root-knot nematodes (Meloidogyne incognita, RKN) and reniform nematodes (Rotylenchulus reniformis, RN) cause the highest yield loss in the U.S. Planting disease resistant cultivars is the most cost effective control method. Numerous studies have reported mapping of quantitative trait loci (QTLs) for disease resistance in cotton; however, very few reliable QTLS were identified for use in genomic research and breeding.

Results: This study first performed a 4-year replicated test of a backcross inbred line (BIL) population for WW resistance, and 10 resistance QTLs were mapped based on a 2895 cM linkage map with 392 SSR markers. The 10 W QTLs were then placed to a consensus linkage map with other $182 \mathrm{WW}$ QTLs, 75 RKN QTLs, 27 FW QTLs, and 7 RN QTLs reported from 32 publications. A meta-analysis of QTLs identified 28 QTL clusters including 13, 8 and 3 QTL hotspots for resistance to WW, RKN and FW, respectively. The number of QTLS and QTL clusters on chromosomes especially in the A-subgenome was significantly correlated with the number of nucleotide-binding site (NBS) genes, and the distribution of QTLs between homeologous A- and D- subgenome chromosomes was also significantly correlated.

Conclusions: Ten WW resistance QTL identified in a 4-year replicated study have added useful information to the understanding of the genetic basis of WW resistance in cotton. Twenty-eight disease resistance QTL clusters and 24 hotspots identified from a total of 306 QTLs and linked SSR markers provide important information for marker-assisted selection and high resolution mapping of resistance QTLs and genes. The non-overlapping of most resistance QTL hotspots for different diseases indicates that their resistances are controlled by different genes.
\end{abstract}

Keywords: Cotton, Verticillium wilt, Fusarium wilt, Root-knot nematodes, Reniform nematodes, Resistance, Quantitative trait loci, Meta-analysis

\footnotetext{
* Correspondence: jinzhang@nmsu.edu; yujw666@hotmail.com

'Department of Plant and Environmental Sciences, New Mexico State University, Las Cruces, NM 88003, USA

${ }^{2}$ State Key Laboratory of Cotton Biology, Institute of Cotton Research of China, Chinese Academy of Agricultural Science, Anyang, Henan 455000,

China

Full list of author information is available at the end of the article
} International License (http://creativecommons.org/licenses/by/4.0/), which permits unrestricted use, distribution, and reproduction in any medium, provided you give appropriate credit to the original author(s) and the source, provide a link to the Creative Commons license, and indicate if changes were made. The Creative Commons Public Domain Dedication waiver (http://creativecommons.org/publicdomain/zero/1.0/) applies to the data made available in this article, unless otherwise stated. 


\section{Background}

Upland cotton (Gossypium hirsutum L., $2 \mathrm{n}=4 \mathrm{x}=52$ ), as a tetraploid cotton, produces $97 \%$ of lint fibers for the textile industry in the world, while extra-long staple (ELS) cotton (G. barbadense L., $2 \mathrm{n}=4 \mathrm{x}=52$ ), also known as Sea-Island, American Pima, or Egyptian cotton accounts for about $3 \%$ of the world cotton. However, various diseases cause substantial yield losses in cotton [1-3]. Verticillium wilt (VW), caused by the soil-borne fungus Verticillium dahliae Kleb., and Fusarium wilt (FW), caused by the soil-borne fungus Fusarium oxysporum f. sp. vasinfectum (Atk.) Synd. \& Hans, are two most destructive diseases in cotton production in the world. VW and FW can significantly reduce cotton yield and fiber quality due to leaf chlorosis, necrosis or wilting, leaf and boll abscission and plant death $[4,5]$. Root-knot nematodes [Meloidogyne incognita (Kofoid \& White), RKN] and reniform nematodes (Rotylenchulus reniformis Linford \& Oliveira, RN) cause the highest yield loss (4.3 and $2.5 \%$, respectively) in the U.S. [3]. Planting disease resistant cultivars is the most effective and economical control method.

Many G. barbadense genotypes are known to carry high levels of resistance to VW [6-8], but its resistance has not been successfully transferred into commercial Upland cotton due to hybrid breakdown except for introgressed breeding lines [7,9]. There have been many studies indicating that the VW resistance in G. barbadense is controlled by a dominant or partially dominant gene in interspecific crosses between G. barbadense and G. hirsutum. In several recent studies, more than 100 VW resistance quantitative trait loci (QTLs) in the interspecific Upland $\times$ Pima populations and also intra- Upland populations have been detected on almost all of the 26 tetraploid cotton chromosomes (c1 through c26) except for $\mathrm{c} 2, \mathrm{c} 6, \mathrm{c} 10, \mathrm{c} 12$, and $\mathrm{c} 18$, and VW resistance QTLs were more frequently detected on c5, c7, c8, c11, c16, c17, c19, c21, c23, c24, and c26 (for a review, see [4]).

A number of qualitative genetic studies have identified five major resistance genes against FW in Upland (in the U.S. and China) and Pima (in the U.S., Egypt and Israel) including $F w 1, F w 2, F w^{R}$ (c17), FOV1 (c16) and FOV4 (c14). There are also numerous quantitative genetic studies using early segregating populations confirming the predominant presence of additive gene effects with low heritabilities on FW resistance. Several recent mapping studies have collectively detected approximately 40 QTLs on all the 26 tetraploid cotton chromosomes except for c1, c4, c5, c10, c13, c20 and c24 (for a review, see [10]).

In Upland cotton, high RKN resistance was achieved in Auburn 623RKN through crossing between moderately resistant Clevewilt and Wild Mexico Jack Jones, which was then transferred to other breeding lines [11]. The resistance was later determined to be controlled by two genes through a classic genetic analysis [12], and the two genes (Mi1 or $q M i-C 11$ and $M i 2$ or $q M i-C 14$ ) were mapped to chromosomes c11 from Clevewilt and c14 from Wild Mexico Jack Jones through a collective research effort $[13,14]$. In an interspecific recombinant inbred line population of two susceptible parents of Upland and Pima cotton, major QTLs (on c3, c4, c11, c14, c17 and c23) and 19 putative QTLs for RKN responses were reported [15].

For $\mathrm{RN}$, the high level of resistance (essentially immunity) in G. longicalyx was transferred to Upland cotton [16], and the resistance is conferred by a single dominant gene $\mathrm{Ren}^{\text {lon }}$ located on chromosome c11 [17]. Moderate resistance was also found in several G. barbadense accessions including GB 713 [18]. Three resistance QTLs (2 on c21 and 1 on c18) were identified in GB 713 in a cross with Acala Nem-X using SSR markers [19], and one of the two QTLs on c21 was later identified as a major QTL through SNP mapping [20]. In a cross between a tri-species hybrid G. arboreum $\times(G$. hirsutum $\times$ G. aridum) and Upland MD51ne, a major dominant resistance gene $\operatorname{Ren}^{\text {ari }}$ also on $\mathrm{c} 21$ presumably from G. aridum was identified using SSR markers [21].

The consistency and utility of most resistance QTLs in breeding and genomic research identified for the above four major diseases remain uncertain. Because most QTL studies used early segregating populations such as $\mathrm{F}_{2}, \quad \mathrm{BC}_{1} \mathrm{~F}_{1}$ and $\mathrm{F}_{2: 3}$, disease resistance could not be repeatedly evaluated for the same genotypes. However, QTLs as reported from different studies provide a good opportunity to perform a meta-analysis of resistance QTLs for identification of consistent resistance QTLs for the same disease (hotspots) and common QTLs for different diseases (clusters) across different studies. QTL clusters for resistance to different diseases and resistance QTL hotspots for the same disease will be very useful for breeders and geneticists to choose chromosome regions for marker-assisted selection and high resolution mapping of disease resistance QTLs or genes.

It is currently known that plant disease resistance is often conferred by disease resistance (R) genes including predominantly nucleotide-binding site (NBS)- encoding genes [22, 23]. $\mathrm{R}$ genes have evolved and clustered on the plant genome through various mechanisms such as tandem and segmental gene duplications, recombination, unequal crossing-over, point mutations, and diversifying selection. Recent genome sequencing studies have identified 391 and 280 NBS-encoding genes in G. raimondii and G. arboreum, respectively [24-26]. However, the relationship between the distribution of the NBS genes and resistance QTLs or genes is unclear in cotton. 
Table 1 QTL for Verticillium wilt (VW) resistance detected in a backcross inbred line (BIL) population of 146 lines derived from a cross of (SG $747 \times$ Giza 75$) \times S G 747 \mathrm{BC}_{2} \mathrm{~F}_{4}$

\begin{tabular}{llllllll}
\hline Year & QTL name & Position (cM) & Marker interval & LOD & Add. & PVE (\%) & Direction \\
\hline 2006 & qWWI-06-c2-1 & 2 & NAU3775a-BNL0663 & 3.32 & 6.23 & 9.90 & SG 747 \\
2008 & qWWI-08-c2-1 & 27 & NAU3684-BNL3971 & 2.93 & -8.59 & 32.38 & Giza 75 \\
2008 & qWWR-08-c4-1 & 7 & BNL3089-NAU3469 & 3.82 & -0.19 & 35.43 & Giza 75 \\
2008 & qWWI-08-c4-1 & 7 & BNL3089-NAU3469 & 4.19 & -7.95 & 41.98 & Giza 75 \\
2007 & qWWI-07-c9-1 & 59 & NAU3358-NAU5494 & 4.22 & 9.54 & 25.04 & SG 747 \\
2007 & qWWR-06-c12-1 & 16 & CIR272-NAU3401b & 3.25 & 0.21 & 10.15 & SG 747 \\
2009 & qWWI-09-c13-1 & 74 & NAU2730-NAU5110 & 2.83 & -11.56 & 25.07 & Giza 75 \\
2006 & qWWI06-c21-1 & 106 & NAU3341a-NAU3895 & 4.42 & 5.72 & 17.17 & SG 747 \\
2008 & qWWI-08-c22-1 & 87 & BNL0206-NAU3392 & 2.86 & -7.53 & 40.39 & Giza 75 \\
2008 & qWWR-09-c23-1 & 80 & NAU5508-NAU3967 & 2.99 & -0.15 & 14.18 & Giza 75 \\
\hline
\end{tabular}

Add. additive effect, PVE phenotypic variance explained, VWI VW incidence, VWR VW rating, c2, c4, c9, c12, c13, c21, c22 and c23, chromosomes

The objectives of this study were to perform a QTL analysis of VW resistance from multiple years of replicated tests on a backcross inbred line (BIL) population of an interspecific Upland $\times$ Pima cross and a metaanalysis of QTLs and genes resistant to VW, FW, RKN and RN identified and reported previously. Relationships between the resistance QTLs and NBS genes on cotton chromosomes were also analyzed.

\section{Results}

\section{QTL mapping for VW resistance in the BIL population}

In the BIL population of 146 lines tested in four years, we detected a total of 10 QTLs for VW resistance based on a linkage map with 392 polymorphic SSR loci spanning a total genetic distance of 2,895 cM as established by $\mathrm{Yu}$ et al. [27]. The QTLs were distributed on 8 chromosomes (Table 1, Additional file 1). These QTLs included two detected in 2006 and 2007 each, five in 2008 and one in 2009. Two VW-QTLs were located on chromosome c2 within a $25-\mathrm{cM}$ region, while another two QTLs were on c4 in the same region with the same marker interval. Other six QTLs were located on c9, c12, c13, c21, c22, and c23 with one QTL each. The VW resistance QTLs were further mapped onto a consensus map (Fig. 1), which shows that VW resistance QTLs on these eight chromosomes were also reported by others previously.

As shown in Table 1, the LOD scores for these QTLs ranged from 2.83 to 4.42, significant based on permutation tests, and phenotypic variation explained (PVE) by each QTL ranged from 9.9 to $42.0 \%$. Six QTLs that were from the resistance parent Giza 75 decreased Verticillium wilt incidents or severity ratings with higher PVE (14.2-42.0\%), while other four QTLs from the susceptible parent SG 747 increased Verticillium wilt incidents or severity ratings with lower PVE (9.9-25.0\%).

\section{Clusters of disease resistance QTLs}

A total of 306 disease resistance QTLs have been reported including $10 \mathrm{VW}$ resistance QTLs identified in this study (Table 2), with $193 \mathrm{VW}$ resistance QTLs accounting for $63 \%$ of the disease QTLs identified in past studies, followed by RKN (75) and FW (27). Chromosomes c23 carried the most resistance QTLs (36), followed by c7 (25 QTLs), c11 (23 QTLs), c21 (22 QTLs), c5 (21 QTLs), c16 (21 QTLs), and c19 (21 QTLs). Chromosomes c4, c8, c9, and c14 each carried 10-17 QTLs; c3, c6, c13, c15, c17, c20, c22, and c26 each carried 6-10 QTLs; c10 carried no resistance QTLs; and the remaining chromosomes $(\mathrm{c} 1, \mathrm{c} 2, \mathrm{c} 12, \mathrm{c} 18, \mathrm{c} 24$ and c25) carried the least number of QTLs (2-5).

For the distribution of resistance QTLs on homeologous chromosomes, four pairs carried more QTLs including three pairs with similar numbers (c5 with 21 QTLs vs. c19 with 21 QTLs; c7 with 25 QTLs vs. c16 with 21 QTLs; and c11 with 23 vs. c21 with 23). However, c23 carried twice QTLs than its homeologous counterpart c9 (36 vs. 17). Four pairs carried less but with similar numbers of QTLs (c1 with 5 vs. c15 with 8; c3 with 9 vs. c17 with 7; c4 with 10 vs. c22 with 9; and c13 with 6 vs. c18 with 2). Among other five pairs carrying a few QTLs, three A-subgenome chromosomes carried less (c2 with 5 vs. c14 with 14; c10 with none vs. c20 with 7; and c12 with 3 vs. c26 with 7); and two other A-subgenome chromosomes carried more (c6 with 8 vs. c25 with 2; and c8 with 14 vs. c24 with 4 ). However, the number of QTLs carried between homeologous chromosomes was significantly and positively correlated $(r=0.682 ; 0.01<P<0.05)$. Overall, the Asubgenome carried 146 QTLs, which was slightly fewer than the D-subgenome (160). However, the difference was not statistically significant $\left(\chi^{2}=0.64 ; \chi_{0.05}^{2}=3.84\right.$ at $\left.\mathrm{df}=1\right)$.

Based on the selection criterion that 4 QTLs within a 25-cM region constitute a cluster, 28 QTL clusters (12 


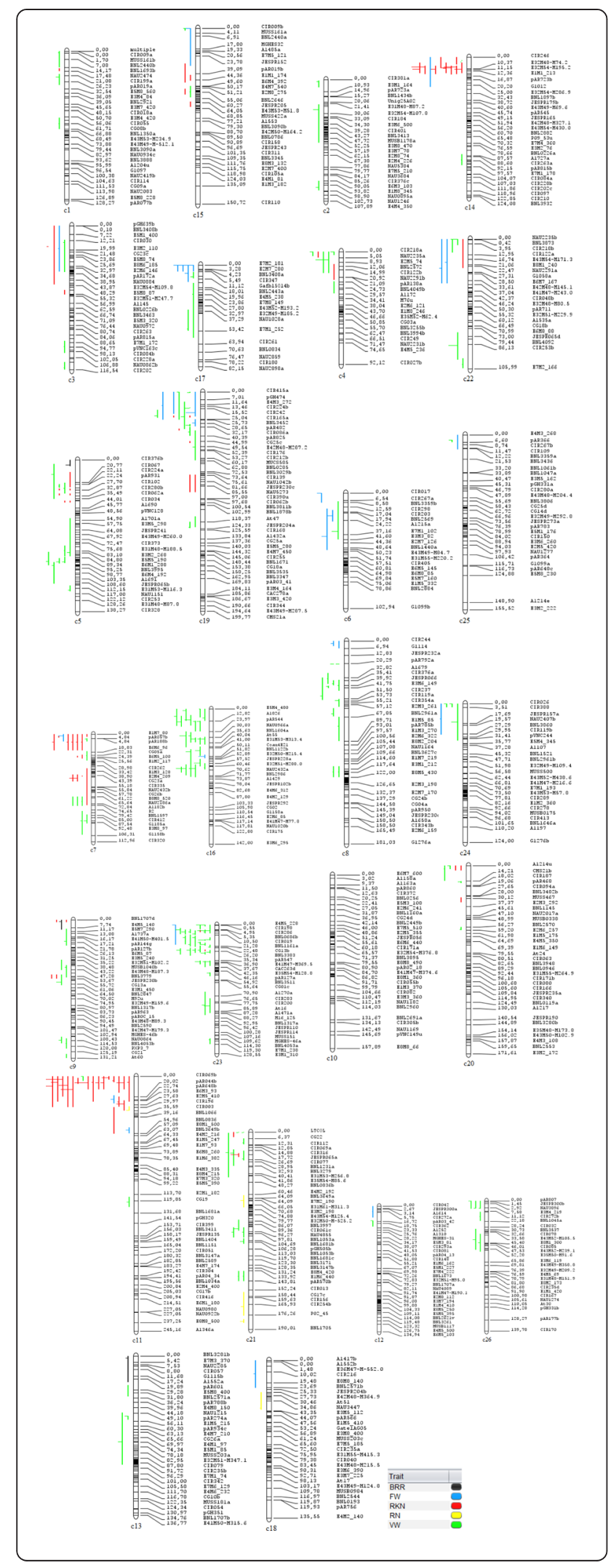

Fig. 1 A meta-analysis of quantitative trait loci (QTLs) for resistance to Verticillium wilt (VW), Fusarium wilt (FW), root-knot nematodes $(\mathrm{RKN})$, reniform nematodes (RN), and black root rot (BRR). When older markers with multiple positions along a chromosome were used as the sole means to position a QTL and the markers did not appear in the cotton marker database those QTL were not able to be placed on the ("Guazuncho2" (G. hirsutum) x "VH8-4602" (G. barbadense)) map. For this reason some QTL identified from previous studies were not included in this meta-analysis and do not appear on the combined QTL linkage map

on the A subgenome- $\mathrm{c} 1$ to $\mathrm{c} 13$ and 16 on the $\mathrm{D}$ subgeome- c14 to c26) on 18 chromosomes (7 on the A subgenome and 11 on the D subgenome) were identified (Table 3), and they contained 210 resistance QTLs (accounting for $\sim 70 \%$ of the total resistance QTLs

Table 2 Numbers of quantitative trait loci (QTLs) resistant to Verticillium wilt ( $\mathrm{WW}$ ), Fusarium wilt (FW), root-knot nematodes $(\mathrm{RKN})$, reniform nematodes (RN), bacterial blight (BB) and black root rot (BRR) that were identified in this study and reported in other studies

\begin{tabular}{|c|c|c|c|c|c|c|c|}
\hline Chromosome & WW & FW & RKN & RN & BB & BRR & Total \\
\hline$\overline{\mathrm{c} 1}$ & 4 & 0 & 1 & 0 & 0 & 0 & 5 \\
\hline$c 2$ & 5 & 0 & 0 & 0 & 0 & 0 & 5 \\
\hline c3 & 5 & 1 & 3 & 0 & 0 & 0 & 9 \\
\hline c4 & 8 & 0 & 2 & 0 & 0 & 0 & 10 \\
\hline$c 5$ & 12 & 0 & 7 & 0 & 1 & 1 & 21 \\
\hline c6 & 5 & 3 & 0 & 0 & 0 & 0 & 8 \\
\hline C7 & 11 & 1 & 13 & 0 & 0 & 0 & 25 \\
\hline c8 & 12 & 2 & 0 & 0 & 0 & 0 & 14 \\
\hline c9 & 12 & 0 & 4 & 0 & 0 & 1 & 17 \\
\hline $\mathrm{c} 10$ & 0 & 0 & 0 & 0 & 0 & 0 & 0 \\
\hline c11 & 2 & 2 & 18 & 1 & 0 & 0 & 23 \\
\hline $\mathrm{c} 12$ & 2 & 1 & 0 & 0 & 0 & 0 & 3 \\
\hline $\mathrm{c} 13$ & 5 & 0 & 0 & 0 & 0 & 1 & 6 \\
\hline c14 & 3 & 1 & 9 & 0 & 1 & 0 & 14 \\
\hline c15 & 3 & 1 & 4 & 0 & 0 & 0 & 8 \\
\hline c16 & 20 & 1 & 0 & 0 & 0 & 0 & 21 \\
\hline $\mathrm{C} 17$ & 3 & 4 & 0 & 0 & 0 & 0 & 7 \\
\hline c18 & 0 & 1 & 0 & 1 & 0 & 0 & 2 \\
\hline C19 & 11 & 4 & 6 & 0 & 0 & 0 & 21 \\
\hline$c 20$ & 4 & 0 & 3 & 0 & 0 & 0 & 7 \\
\hline C21 & 16 & 0 & 2 & 5 & 0 & 0 & 22 \\
\hline$c 22$ & 6 & 2 & 1 & 0 & 0 & 0 & 9 \\
\hline c23 & 33 & 1 & 2 & 0 & 0 & 0 & 36 \\
\hline$c 24$ & 3 & 1 & 0 & 0 & 0 & 0 & 4 \\
\hline$c 25$ & 1 & 1 & 0 & 0 & 0 & 0 & 2 \\
\hline$c 26$ & 7 & 0 & 0 & 0 & 0 & 0 & 7 \\
\hline Total & 193 & 27 & 75 & 7 & 2 & 3 & 306 \\
\hline
\end{tabular}


Table 3 Resistance QTL clusters and hotspots identified for Verticillium wilt (WW), Fusarium wilt (FW), rootknot nematodes (RKN) and reniform nematodes (RN)

\begin{tabular}{|c|c|c|c|c|c|c|}
\hline Chr & Total & No. clusters & No. QTLS & Type hotspot & Region & Linked markers \\
\hline$\overline{\mathrm{Cl}}$ & 5 & 0 & & & & \\
\hline$c 2$ & 5 & 0 & & & & \\
\hline c3 & 9 & 0 & & & & \\
\hline c4 & 10 & 1 & $5 \mathrm{WW}, 2$ RKN & WW & $0-10 \mathrm{cM}$ & CIR 210A, NAU2235a, BNL2572CIR122B, NAU2291B \\
\hline c5 & 21 & 2 & 7 RKN, 3 WW, 1 BRR & RKN & $10-35 \mathrm{cM}$ & CIR067, CIR224a, CIR102, CIR280b, CIR062a \\
\hline c5 & & & $5 \mathrm{WW}$ & WW & $75-100 \mathrm{cM}$ & CIR373, BNL3995, JESPR065b \\
\hline$c 6$ & 8 & 1 & $5 \mathrm{FW}, 1 \mathrm{WW}$ & FW & $5-25 \mathrm{Cm}$ & CIR267a, BNL3359b, CIR298, CIR203, BNL2569 \\
\hline C7 & 25 & 3 & $10 \mathrm{RKN}, 2 \mathrm{WW}, 1 \mathrm{FW}$ & RKN & $0-25 \mathrm{Cm}$ & pAR057b, pAR188b, CG05a \\
\hline C7 & & & 4 RKN & RKN & $40-55$ cM & CG23a, CIR355, NAU2432b \\
\hline C7 & & & $5 \mathrm{WW}$ & WW & $65-85 \mathrm{cM}$ & NAU2186a, R2, BNL1597, CIR412 \\
\hline c8 & 14 & 2 & $2 \mathrm{FW}, 2 \mathrm{WW}$ & & $0-20 \mathrm{cM}$ & CIR244, G1114, JESPR232a, pAR792a \\
\hline C8 & & & $7 \mathrm{WW}$ & WW & $35-60 \mathrm{Cm}$ & CIR376a, JESPR066, CIR237, CIR254a, BNL2961a \\
\hline c9 & 17 & 1 & $3 \mathrm{WW}, 2$ RKN & & $35-55 \mathrm{cM}$ & MUSB1040b, BNL3799, JESPR230b \\
\hline c10 & 0 & 0 & & & & \\
\hline $\mathrm{c} 11$ & 23 & 2 & $17 \mathrm{RKN}, 1 \mathrm{WW}, 1 \mathrm{FW}$ & RKN & $0-25 \mathrm{cM}$ & CIR069b, pAR044b, pAR648b \\
\hline $\mathrm{c} 11$ & & & 4 RKN, 2 FW, 1 RN ${ }^{a}$ & RKN & $20-45 \mathrm{cM}$ & pAR044b, pAR648b, CIR196, CIR003, BNL1066, BNL0836 \\
\hline $\mathrm{c} 12$ & 3 & 0 & & & & \\
\hline $\mathrm{c} 13$ & 6 & 0 & & & & \\
\hline c14 & 14 & 1 & 9 RKN, 1FW & RKN & $0-20 \mathrm{cM}$ & CIR246, pAR723b, G1012 \\
\hline c15 & 8 & 1 & $4 \mathrm{RKN}, 1 \mathrm{WW}$ & RKN & $40-60 \mathrm{cM}$ & pAR015b, BNL2646, JESPR205 \\
\hline c16 & 21 & 3 & $8 \mathrm{WW}$ & WW & $0-25 \mathrm{cM}$ & A1826, pAR544 \\
\hline c16 & & & $6 \mathrm{WW}, 1 \mathrm{FW}$ & WW & $30-55$ cM & HAU0966a, BNL1604a, BNL1122b \\
\hline c16 & & & $6 \mathrm{WW}$ & WW & $57-75 \mathrm{cM}$ & JESPR228a, HAU2432a, BNL2986 \\
\hline $\mathrm{c} 17$ & 7 & 1 & $4 \mathrm{FW}, 2 \mathrm{WW}$ & FW & $0-10 \mathrm{cM}$ & BNL3408a, CIR347, BNL2443a \\
\hline $\mathrm{c} 18$ & 2 & 0 & & & & \\
\hline c19 & 21 & 2 & $5 \mathrm{WW}, 5 \mathrm{FW}$ & WW, FW & $0-25 \mathrm{cM}$ & CIR415a, CIR224b, CIR242, CIR165a \\
\hline c19 & & & $4 \mathrm{RKN}, 2 \mathrm{WW}$ & RKN & $20-50 \mathrm{cM}$ & CIR165a, BNL3452, CIR086a, CIR176 \\
\hline $\mathrm{c} 20$ & 7 & 1 & $3 \mathrm{WW}, 2$ RKN & & $0-20 \mathrm{cM}$ & A1214a, CMS21b, CIR187 \\
\hline$c 21$ & 22 & 2 & $5 \mathrm{WW}, 2$ RKN, 2 FW & WW & $0-25 \mathrm{cM}$ & LTCOL, CG22, CIR112, CIR069a, CIR316, JESPR066a \\
\hline$c 21$ & & & $4 \mathrm{WW}$ & & $85-105 \mathrm{cM}$ & BNL3997, CIR061c, HAU4855, BNL1053a, BNL1681b \\
\hline$c 22$ & 9 & 1 & $2 \mathrm{WW}, 2 \mathrm{FW}, 1 \mathrm{RKN}$ & & $0-25 \mathrm{cM}$ & NAU2235b, BNL3873, CIR218b, CIR122a, NAU2291a \\
\hline$c 23$ & 36 & 2 & $22 \mathrm{WW}, 1 \mathrm{FW}, 2 \mathrm{RKN}$ & WW & $0-25 \mathrm{cM}$ & CIR198, CIR286, BNL086b, CIR019, BNL1161a, BNL3383 \\
\hline$c 23$ & & & $4 \mathrm{WW}$ & WW & $35-45 \mathrm{cM}$ & pAR517, CAC263d \\
\hline$c 24$ & 4 & 1 & $3 \mathrm{WW}, 1 \mathrm{FW}$ & & $0-25 \mathrm{cM}$ & CIR026, CIR388, JESPR157a, HAU2407b, BNL3860 \\
\hline$c 25$ & 2 & 0 & & & & \\
\hline$c 26$ & 7 & 1 & $4 \mathrm{WW}$ & WW & $0-20 \mathrm{cM}$ & JESPR300b, NAU3896, CIR272b, BNL1045a \\
\hline
\end{tabular}

BRR black root rot
a a major resistance gene Ren $^{\text {Ion }}$ transferred to Upland from G. longicalyx

identified). The difference in distribution of clusters on the A- and D- subgenomes was not significant $\left(\chi^{2}=\right.$ $0.57 ; \chi_{0.05}^{2}=3.84$ at $\left.\mathrm{df}=1\right)$. The first region $($ at $0-25 \mathrm{cM})$ on the chromosomes of the cotton genome contained most QTL clusters (16), followed by the second region at 25-50 cM with 8 clusters; and the third region at 60-85
$\mathrm{cM}$ and the fourth region at 75-100 cM only carried 2 clusters each. Across the cotton genome, the difference in distribution of clusters in the four regions was highly significant $\left(\chi^{2}=18.86 ; \chi_{0.05}^{2}=7.82\right.$ and $\chi_{0.01}^{2}=16.27$ at $\left.\mathrm{df}=3\right)$. There were no clusters on c1, c2, c3, c10, c12, c13, c18, and c25. Chromosomes $\mathrm{c} 7$ and c16 each carried 3 clusters, 
while c5, c8, c11, c19, c21, and c23 each carried 2 clusters. However, some of the clusters did not contain any QTL hotspot (at least 4 resistance QTLs for the same disease) including $\mathrm{c} 8$ (2 VW and $2 \mathrm{FW}$ QTLs at $0-20 \mathrm{cM}), \mathrm{c} 9$ (3 VW and 2 RKN QTLs at 35-55 cM), c20 (3 VW and 2 RKN QTLs at $0-20 \mathrm{cM}), \mathrm{c} 22$ (2 VW, $2 \mathrm{FW}$ and 1 RKN QTL at $0-25 \mathrm{cM}$ ) and $\mathrm{c} 24$ ( $3 \mathrm{VW}$ and $1 \mathrm{FW}$ QTL at 0-20 cM). Some of the clusters may contain QTL hotspots after more QTL mapping results are reported in the future. In the following sections, clusters containing resistance QTL hotspots for each disease will be analyzed in more details.

Comparing the distribution of QTL clusters between homeologous chromosomes (Table 3), c1, c2, c3, c10, and $\mathrm{c} 12$ did not have any clusters identified, while their counterparts (c15, c14, c17, c20 and c26, respectively) each had one cluster. However, c6 had one cluster while its homeologous chromosome c25 carried no cluster, nor the homeologous pair c13 and c18 carried any clusters. Homeologous chromosome pair c4 and c22 each carried one cluster in similar regions with QTLs for VW and RKN resistance identified, while other three pairs (c5 vs. c19, c7 vs. c16, and c11 vs. c21) each carried 2-3 clusters. For c5 and c19, 3-5 VW QTLs were identified in the similar region $(0-30 \mathrm{cM})$, while the second cluster differed in map positions and types of QTLs between the two chromosomes. For c7 and c16, the first cluster had 2 VW QTLs; the second cluster differed in QTL types, while the third cluster had the same QTL type but differed in positions. For c11 and c21, the first cluster in a similar position only had 2 QTLs in common for the same traits, while the second cluster differed in QTL type and position. Interestingly, for homeologous pair c9 and c23, both had 3 VW QTLs in a similar region (30$50 \mathrm{cM}$ ). However, c23 carried 2 clusters (with 25 and 4 QTLs, respectively), while c9 carried one cluster with only 5 QTLs. For another homeologous pair c8 (with 2 clusters) and c24 (with one), both had QTLs for VW and FW resistance in the same region $(0-20 \mathrm{cM})$.

\section{Meta-analysis of Verticillium wilt (VW) and Fusarium wilt (FW) resistance QTLs}

Of a total of 193 QTLs for VW resistance, 83 and 110 were distributed on the A- and D- subgenomes, respectively (Table 2, Fig. 1). But the difference in QTLs between the two subgenomes was not statistically significant $\left(\chi^{2}=3.78 ; \chi_{0.05}^{2}=3.84\right.$ at $\left.\mathrm{df}=1\right)$. Except for chromosomes c10 and c18, all chromosomes carried VW resistance QTLs. Chromosome c23 carried the most QTLs (33), followed by c16 with 20 and c21 with 16 . Chromosomes c5, c7, c8, c9 and c19 each carried 11-12 QTLs, while c4 and c26 carried 7-8 QTLs. Due to the concentration of QTLs in regions, c4 (at $0-10 \mathrm{cM}), \mathrm{c} 5$ (at $80-100 \mathrm{cM}), \mathrm{c} 7$ (at $65-85 \mathrm{cM}), \mathrm{c} 8$ (35-55 cM), c19 (at $0-25 \mathrm{cM}$ ), and c26 (at $0-20 \mathrm{cM}$ ) each carried one hotspot with 4-7 VW resistance QTLs, while c21 and c23 each carried 2 hotspots, and c16 carried 3 hotspots (Table 3). On c16, the three hotspots were distributed at $0-20,30-40$, and 50-65 cM with 8, 6 and 6 QTLs, respectively. On $\mathrm{c} 21$, at two distal regions (at $0-$ $25 \mathrm{cM}$ and 85-105 cM) from the centromere, the two hotspots each contained 4-5 QTLs. On c23, the hotspot at $0-25 \mathrm{cM}$ had 22 QTLs concentrated for VW resistance, while the one at 35-45 cM had only 4 VW resistance QTLs. The above 13 hotspots contained 86 individual VW resistance QTLs (45\% of total VW QTLs) and a few QTLs for FW or RKN resistance. Of the $193 \mathrm{VW}$ resistance QTLs identified, 111 (57 \%) were located in disease resistance QTL clusters.

All 27 FW resistance QTLs reported on 16 chromosomes were located in resistance QTL clusters, 14 of which were located on three chromosomes c6, c17 and c19 (Table 2, Fig. 1). The relevant regions $(0-25 \mathrm{cM})$ on these three chromosomes (5 QTLs at 5-25 cM on c6, 4 QTLs at $0-10 \mathrm{cM}$ on $\mathrm{c} 17$, and 5 QTLs at $0-25 \mathrm{cM}$ on c19) each carried a QTL hotspot for FW resistance (Table 3). It is interesting to note that the same region on c19 also carried a QTL hotspot with 5 QTLs for VW resistance. Therefore, this region may have resistance genes for both VW and FW.

\section{Meta-analysis of root-knot nematode (RKN) and reniform nematode (RN) resistance QTLs}

For RKN resistance, a total of 75 QTLs were identified on 14 chromosomes (Table 2, Fig. 1), 18 of which were located on c11, followed by c7 (13 QTLs), c14 (9 QTLs), c5 (7 QTLs), c19 (6 QTLs), c9 (4 QTLs), and c15 (4 QTLs). Chromosomes c1, c3, c4, c20, c21, c22 and c23 each carried 1-3 RKN resistance QTLs, and the remaining chromosomes did not carry any RKN resistance QTLs. Eight QTL hotspots were identified including two each on $\mathrm{C} 7$ and $\mathrm{c} 11$ (at 0-25 and 20-45 cM regions), and one each on c5 (at 10-35 cM), c14 (at 0-20 cM), c15 (at 40-60 cM), and $\mathrm{c} 19$ (at 20-50 cM). Two major RKN resistance genes or QTLs with major effects from Auburn 623RKN and its derived lines, as reported previously $[13,14]$, have been confirmed in the hotspot regions of c11 with 17 QTLs and c14 with 9 QTLs. Of the 75 RKN QTLs identified, 69 (92\%) and 59 (79\%) were located within 14 resistance QTL clusters and the 8 RKN resistance QTL hotspots, respectively (Table 3).

Of the six QTLs reported for reniform nematode (RN) resistance, c21 carried five, while c18 carried one. Since the five QTLs on c21 were scattered along the whole chromosome, there was no QTL hotspot identified. 
However, c11 carried one major dominant resistance gene $\operatorname{Ren}^{\text {lon }}$ transferred from G. longicalyx, and it was in a close proximity to the two RKN resistance QTL hotspots with 21 QTL identified including one of the two major resistance genes for RKN resistance (Table 3, Fig. 1). Therefore, this c11 region carries important resistance genes for both RKN and RN resistance.

\section{QTLs for resistance to bacterial blight (BB) and black root rot (BRR)}

Due to the RFLP markers used by Wright et al. [28], the $\mathrm{BB}$ resistance QTLs could not be placed on the consensus map in this study. However, three reported QTLs resistant to BRR were mapped onto $\mathrm{c} 5, \mathrm{c} 9$ and $\mathrm{c} 13$ of the consensus map (Fig. 1). The one on c5 was close to a QTL cluster with 7 RKN and 3 VW QTLs, while the other two QTLs were distant from others.

\section{Identification of linked markers to the disease resistance QTL clusters and hotspot}

The closely linked SSR markers for the 28 disease resistance QTL clusters involving $13 \mathrm{VW}$ resistance hotspots, 3 FW resistance hotspots and 7 RKN resistance hotspots are listed in Table 3. The information should be useful to breeders and geneticists.

\section{Association between number of resistance QTLs and number of nucleotide-binding site (NBS)-encoding genes} Based on the sequenced genomes in G. raimondii (D5 genome) and G. arboreum (A2 genome), 391 and 280 NBS-coding genes were identified, respectively [24]. The significantly higher number of NBS genes in G. raimondii than in G. arboreum $\left(\chi^{2}=18.36 ; \chi_{0.05}^{2}=3.84\right.$ at $\left.\mathrm{df}=1\right)$ may explain why the $\mathrm{D}$ subgenome carried more disease resistance QTLs than the A subgenome in the tetraploid cotton. The number of NBS genes and the number of QTLs identified on the $13 \mathrm{D}$-subgenome chromosomes were positively correlated $(r=0.399 ; P>0.05)$, while the correlation was significant with the number of total disease resistance QTLs on homeologous A-subgenome chromosomes $(r=0.645 ; 0.01<P<0.05)$. The sum of QTLs in 13 pairs of homeologous chromosomes was also significantly correlated with the number of NBS-coding genes $(r=0.553 ; 0.01<P<0.05)$. The correlation with the number VW QTLs on A- and D- subgenome chromosomes was also positive but insignificant $(r=0.387-0.464$; $P>0.05$ ); however, the correlation with the number of RKN QTLs on the A subgenome chromosomes was significant $(r=0.645 ; 0.01<P<0.01)$. Therefore, there is a trend that the more NBS-encoding genes a chromosome carries, the more disease resistance QTLs it has. For example, chromosome 7 (D5) carried the most number of NBS genes (87), and its homeologous pair of Upland cotton (c7 vs. c16) carried a total of 46 QTLs (25 vs. 21$)$.
Chromosomes 9 and 11 in D5 carried 23 and 34 NBS genes, respectively, and their homeologous pairs (c9 vs. c23, and c11 vs. c21) carried a total of 53 (17 vs. 36) and 46 (23 vs. 23) QTLs, respectively. Chromosomes 6, 10, 12 and 13 in D5 contained a minimum numbers of NBS genes (1-8), their corresponding tetraploid chromosomes (c6 vs. c25, c10 vs. c20, c12 vs. c26, and c13 vs. c18) also carried the least numbers of QTLs ( 8 vs. 2, 0 vs. 7,3 vs. 7 , and 6 vs. 2, respectively). However, three chromosomes (1,2, and 8) in D5 also had high numbers of NBS genes $(24,22$, and 32, respectively), but their tetraploid counterparts (c1 vs. c15, c2 vs. c14, and c8 vs. c24, respectively) only carried moderate numbers of QTLs ( 5 vs. 8,5 vs. 14 , and 14 vs. 4 , respectively). Surprisingly, chromosomes 5 in D5 carried a small number of NBS genes (5), but its tetraploid counterparts (c5 vs. c19) carried a high number of QTLs (21 vs. 21).

Furthermore, the number of NBS genes was significantly correlated with the number of QTL clusters on $\mathrm{A}$ - and D- subgenome chromosomes and the sum of the homeologous chromosomes $(r=0.683, r=0.710$, and $r=$ 0.754, respectively; $\mathrm{r}_{0.05}=0.553, \mathrm{r}_{0.01}=0.684 ; \mathrm{df}=11$ ). For example, chromosomes 7, 8, 9 and 11 with high numbers of NBS genes each had a total of 3-6 clusters in their tetraploid counterparts. Since NBS genes on these chromosomes with high numbers of NBS genes are clustered, the results indicate that the disease resistance QTL clusters may be in part determined by NBS gene clusters. However, the reverse is true for chromosome 5 with only 5 NBS genes in the D5 genome, while its tetraploid counterparts carried 4 clusters. This result indicates that either these NBS genes may have pleiotropic effects on multiple diseases or other genes on the chromosome are involved in quantitative resistance.

\section{Discussion}

\section{Difficulties in screening cotton for $\mathrm{VW}$ resistance}

In this study, a BIL population of 146 lines was tested in 2-4 replications (with 27-30 plants for each genotype in each replication) through 4-years replicated field tests. Disease resistance was determined by an average severity rating from 54-120 individual plants for each genotype, therefore rendering low experimental errors than most previous VW resistance studies. However, only $10 \mathrm{VW}$ resistance QTLs on 8 chromosomes were identified. On two chromosome regions, two QTLs were identified in the same or similar regions. On the 8 chromosomes where the 10 QTLs were mapped, VW resistance QTLs were also mapped by previous studies. In fact, 4 QTLs were mapped onto three chromosomes (c4, c21 and c23) with VW QTL hotspots. Therefore, the results in this study demonstrated a moderate level of consistency in QTL mapping for VW resistance based on the multiple 
years of replicated studies. The results also demonstrated difficulties in VW resistance studies even if inbred lines are evaluated for VW resistance in multiple replicated field tests with or without inoculations.

As Zhang et al. $[4,10]$ noted, it is difficult to reliably identify VW and FW resistance QTLs. There are a number of contributing factors to the complication of disease resistance studies. First, Many early segregating populations were used in disease resistance QTL mapping, which did not allow repeated evaluation of the same genotypes from multiple individuals in multiple replications and multiple environments. Experimental errors were understandably higher. Second, VW and FW disease infections are highly sensitive to environmental and developmental factors, and even artificial inoculations could not achieve similar disease infections in the same genotypes $[4,8,29,30]$. Thirdly, an artificial grading system for disease severity is often used, rendering it very difficult to quantitatively and accurately phenotype cotton responses to the diseases. Fourth, there exist interactions of genotype with environment, strains of a pathogen, and evaluation methods, resulting in different disease responses of the same genotypes to different strains of pathogens under different environmental conditions or using different inoculation methods. Furthermore, most disease resistance QTLs have low contributions to disease resistance, which could not be detected in many environmental conditions where experimental errors for resistance screening are higher. Finally, low genome coverage of molecular markers in many mapping studies does not allow a genome-wide detection of QTLs with a high resolution, resulting in different QTLs identified from different genetic populations evaluated under different environmental and screening conditions. All of these issues call for reliable screening techniques and phenotyping of disease resistance in mapping populations with multiple individuals in each genotype using multiple replicated tests. Of course, genome-wide markers should be developed for better genome coverage, so more QTLs with high accumulated PVE will be detected.

\section{Genetic basis of VW resistance}

The complexity in mapping QTLs for VW resistance in cotton is further illustrated from the meta-analysis of VW resistance QTLs reported previously. Out of 193 QTLs reported, except for $\mathrm{c} 10$ and $\mathrm{c} 18$ where no QTLs for VW resistance were reported, all other chromosomes had QTLs mapped, although 14 chromosomes carried only 1-6 VW QTLs. The identification of 13 VW resistance QTL hotspots on 9 chromosomes further demonstrated the complexity in studying the genetic basis of VW resistance in cotton, because selection of QTLs for
VW resistance breeding and further genetic and genomic studies will be difficult. However, more attention should be paid to several VW QTL populated hotspots, such as the hotspot with 7 VW QTLs on c8 at 35$60 \mathrm{cM}$ and three hotspots with $22 \mathrm{VW}$ QTLs on c16 at 0-75 cM. But, the most notable is the hotspot with 22 VW QTLs on c23 at $0-25 \mathrm{cM}$, because this region may also confer resistance to FW and RKN since 1-2 resistance QTLs to FW and RKN were identified.

Several other regions also deserve more attention. The VW QTL hotspot on c19 is interesting, because a FW QTL hotspot was within the same region, and a RKN QTL hotspot was also in the proximity. Therefore, this region may share genes responsive to $\mathrm{VW}, \mathrm{FW}$ and RKN.

A region on c11 is also very important in conferring resistance to multiple diseases. In a broad region (0-45 cM), 21 RKN QTLs, 3 FW QTLs, 1 VW QTL, and 1 major $\mathrm{RN}$ resistance gene $\left(\mathrm{Ren}^{\text {lon }}\right)$ were mapped. Another region $(0-20 \mathrm{cM})$ with the second most frequent RKN QTLs was on c14. In this region, a major gene or QTL was identified for RKN resistance [13, 14]. However, this region appeared to confer resistance to only RKN, because there were very few QTLs identified for resistance to other diseases.

Chromosome c21 also deserves more consideration, because it carried two QTL hotspots for VW resistance, 2 QTL for RKN resistance and also 5 QTLs for reniform nematode (RN) resistance. However, the RN resistance QTLs were scattered along the chromosome and not contained in any of the hotspots. Except for the hotspot in the same region on $\mathrm{c} 19$ for VW and FW resistance, other hotspots including 8 hotspots for RKN resistance did not overlap with any of the resistance QTL hotspots for VW and FW. In fact, the numbers of QTLs for VW, FW and RKN on different chromosomes were not correlated $\left(r=-0.039\right.$ to $0.114 ; \mathrm{r}_{0.05}=0.388$ at $\left.\mathrm{df}=24\right)$. Therefore, it is reasonable to speculate that the resistance mechanisms for the three diseases are likely different. However, the linked markers for 13 VW hotspots, 3 FW hotspots and 8 RKN hotspots should be highly useful in choosing chromosome regions with consistent QTLs for marker-assisted selection and high resolution mapping of resistance QTLs and genes.

\section{NBS-coding genes and disease resistance QTLS}

In rice and other plant species, many disease resistance genes were cloned and isolated [22, 23]. It is known that most of the disease resistance genes belong to a super gene family encoding nucleotide-binding site (NBS) domains. Through resistance gene analog RGA-based marker analysis, many RGAs in cotton were mapped [31-33]. Except that a RGA marker was found to be linked to a RKN resistance gene in a study [32], no other 
studies have associated NBS genes with disease resistance in cotton. Based on the current study, the number of disease resistance QTLs and QTL clusters including hotspots identified on chromosomes seemed to be positively correlated with the number of NBS genes. However, on several other chromosomes with very few NBS genes, substantially higher numbers of resistance QTLs were identified. It is likely that in some QTL cluster and hotspot regions, multiple NBS genes are located, and different NBS genes may confer resistance to different diseases. Since NBS-coding $\mathrm{R}$ genes are normally major Mendelian resistance genes [22, 23], the positive correlation of QTL clusters and hotspots identified in the current meta-analysis with NBS genes indicates that these QTL regions maybe contain major resistance $\mathrm{R}$ genes. In fact, major disease resistance genes or QTLs with major effects have been identified for VW, FW, RKN, RN, and BB [4, 10, 12-14, 17, 19-21, 34]. For example, two major resistance genes were identified for RKN [12-14]; and more than 12 major genes resistant to various races of $\mathrm{BB}$ including one on chromosome 5 have been reported [34]. Others major resistance genes include one resistant to southwestern cotton rust (Puccinia ccabata Arth. and Holw.) and two resistant to cotton leaf crumple virus [34]. Recent studies have shown that some major disease resistance $\mathrm{R}$ genes in other plants are co-localized with resistance QTLs, suggesting weak or defeated effects of $\mathrm{R}$ genes or their tight linkage with other genes responsible for quantitative resistance loci [35]. There are other genes with different functions identified recently that may be responsible for quantitative disease resistance [35, 36]. Further studies are needed to discern the relationship between disease resistance QTLs and NBS genes in cotton. Because a $25 \mathrm{cM}$ region may contain 700-800 genes based on the sequenced diploid cotton genomes, identification of candidate genes for the resistance QTL clusters is currently impractical in this study. In the future, positional candidate gene approaches in relating NBS genes to resistance QTLs will be possible once the tetraploid cotton genome is sequenced and the QTL clusters are narrowed to 5-10 cM regions through a high resolution mapping strategy using large genetic populations.

\section{Materials and methods Materials}

An interspecific backcross inbred line (BIL) population comprising of 146 lines was used in this study. The BILs were developed from a cross between Upland cotton ( $G$. hirsutum) SG 747 and G. barbadense Giza 75 through two generations of backcrossing using SG 747 as the recurrent parent followed by four generations of selfpollination. During the BIL development, each $\mathrm{BC}_{1} \mathrm{~F}_{1}$ plant was used as male parent to backcross with SG 747 to derive $\mathrm{BC}_{2} \mathrm{~F}_{1}$. In each $\mathrm{BC}_{1} \mathrm{~F}_{1}$-derived $\mathrm{BC}_{2} \mathrm{~F}_{5}$ progeny, one single representative plant was selected for seed increase and used as the seed source for subsequent field tests. The 146 BILs and the two parents were planted in the experimental farm of China Cotton Research Institute, Chinese Academy of Agricultural Sciences, Anyang, Henan province in 2006, 2007 and 2008. The fields used to evaluate VW resistance were grown with cotton yearly and heavily infected with race 3 of $V$. dahliae Kleb. To further evaluate VW resistance, an artificially inoculated field nursery with the VW strain from Anyang was used in 2009. The 148 entries were arranged in a randomized complete block design with two (2007), three (2008 and 2009) and four replications (2006). Seeds were sown in single row plots in April and crop managements followed local recommendations. The plot length was $8.3 \mathrm{~m}$ with a row-spacing of $0.8 \mathrm{~m}$ and seedlings were thinned to $27-30$ plants per plot.

\section{VW resistance screening}

All the individual plants in each plot were evaluated for VW resistance based on a system established as a national standard for screening cotton for VW resistance in China [4], as the following,
0 . No symptom (healthy)
1. $<25 \%$ chlorotic/necrotic leaves
2. $25-50 \%$ chlorotic/necrotic leaves
3. $50-75 \%$ chlorotic/necrotic leaves
4. $>75 \%$ chlorotic/necrotic leaves
5. Complete defoliation or plant death

The number of infected plants was divided by the total number of plants screened to calculate disease incidence (VW \%), and average disease severity rating-VWR, i.e., the sum of (rating $\times$ number of plants) was divided by the total number of plants [4,7]. The average disease severity rating on the $0-5$ scale was converted to the disease index (\%) on a $0-100 \%$ scale as the ratio between the average severity rating and the highest rating (i.e., 5).

\section{DNA extraction, maker analysis, and map construction}

The genomic DNAs were extracted from young leaves of the 146 individual BIL lines and the two parents using a quick method [37]. Simple sequence repeat markers (SSRs) were used to construct a genetic map for the BIL population using JoinMap 3.0 [38] and the linkage map was published elsewhere [27].

\section{QTL mapping}

For QTL mapping, the IciMapping software (v3.2; http://www.isbreeding.net/), an integrated software for building linkage maps and mapping QTLs which can 
handle various mapping populations including BILs in this study, was used [39]. See Yu et al. [27] for details. The QTL nomenclature followed McCouch et al. [40] in that a QTL designation begins with "q", followed by an abbreviation of the trait name, year, chromosome name, and finally a serial number.

\section{Meta-analysis of QTLs}

As of the end of January 2015, results of disease resistance QTL mapping from all publicly accessible journals were obtained, in addition to a few papers published in
Chinese in China. This study used 32 published papers regarding mapping of resistance to VW, FW, RKN, RN, bacterial blight (BB) and black root rot (BRR) with 306 QTLs reported (see Table 4 for details). For VW resistance QTL mapping, 12 studies [41-50] were included. For FW QTL mapping, 6 studies [51-56] were included. For RKN QTL mapping, 7 studies [13, 14, 57-60] were included. For RN QTL mapping, 5 studies were included [17, 19-21]. To be more inclusive, results from two studies on BB and BRR resistance QTL mapping were also used $[28,61]$.

Table 4 Mapping of resistance to Verticillium wilt (WW), Fusarium wilt (FW), root-knot nematodes (RKN) and reniform nematodes (RN) that were identified in this study and reported in other studies

\begin{tabular}{|c|c|c|c|c|c|}
\hline Author & Journal & Year & No. QTL & Population & Disease \\
\hline Wright RJ et al. & Genetics & 1998 & 2 & F2 & BB \\
\hline Niu C et al. & Theor Appl Genet & 2008 & 3 & $F 2 / F 2: 3$ & BRR \\
\hline Wang \& Roberts & Phytopathology & 2006 & 1 & $F 2: 3$ & FW \\
\hline Wang PZ et al. & Theor Appl Genet & 2009 & 6 & $F 2: 3$ & FW \\
\hline Lopez-Lavalle et al. & Mol Breed & 2012 & 9 & F3/F4 & FW \\
\hline Mei $\mathrm{H}$ et al. & Euphytica & 2014 & 7 & $4 \mathrm{WC}$ & FW \\
\hline Ulloa M et al. & Mol Genet Genomics & 2011 & 6 & RIL & FW \\
\hline Ulloa M et al. & Theor Appl Genet & 2013 & 4 & $F 2 \& R I L$ & FW \\
\hline Gutierrez OA et al. & Theor Appl Genet & 2010 & 12 & RIL & RKN \\
\hline He et al. & Theor Appl Genet & 2014 & 1 & F2 & RKN \\
\hline Shen $X$ et al. & Theor Appl Genet & 2010 & 1 & $F 2$ & RKN \\
\hline Shen $\mathrm{X}$ et al. & Theor Appl Genet & 2006 & 13 & F2 & RKN \\
\hline Wang $C$ et al. & Theor Appl Genet & 2006 & 1 & RIL & RKN \\
\hline Wang C et al. & PLOS ONE & 2012 & 45 & RIL & RKN \\
\hline Ulloa M et al. & Plant Breed & 2009 & 2 & $\mathrm{BC} 1 \mathrm{P} 1 / \mathrm{P} 2$ & RKN \\
\hline Buyyarapu R et al. & ICGI 2014 Conf & 2014 & 1 & F2 & $\mathrm{RN}$ \\
\hline Dighe ND et al. & Crop Sci & 2009 & 1 & BCF1/BCS1 & $\mathrm{RN}$ \\
\hline Gutierrez OA et al. & Theor Appl Genet & 2011 & 3 & $\mathrm{BCP} 1 / 2$ & $\mathrm{RN}$ \\
\hline Romano GB et al. & Theor Appl Genet & 2009 & 1 & Trispecific & $\mathrm{RN}$ \\
\hline Bolek et al. & Plant Sci & 2005 & 33 & F2 & WW \\
\hline Fang $\mathrm{H}$ et al. & Mol Breed & 2014 & 19 & RIL & WW \\
\hline Fang $\mathrm{H}$ et al. & Euphytica & 2013 & 3 & BIL & WW \\
\hline Ge HY et al. & Cotton Sci & 2008 & 1 & $F 2: 3$ & WW \\
\hline Jiang F et al. & Sci in China Ser C: Life Sci & 2009 & 41 & $F 2: 3$ & WW \\
\hline Wang FR et al. & Cotton Sci & 2007 & 4 & $F 2: 3$ & WW \\
\hline Zhao Y et al. & PLOS ONE & 2014 & 14 & AM & WW \\
\hline Wang $P$ et al. & The Crop Journal & 2014 & 23 & CSILS & WW \\
\hline Wang HM et al. & J Integr Plant Biol & 2008 & 4 & $F 2: 3$ & WW \\
\hline Zhang $X$ et al. & PLOS ONE & 2014 & 5 & $F 2: 3$ & WW \\
\hline Yang $C$ et al. & Plant Sci & 2008 & 18 & $\mathrm{BC} 1 \mathrm{~S} 2$ & WW \\
\hline Ning ZY et al. & Crop Sci & 2013 & 12 & RIL & WW \\
\hline Zhang JF et al. & This study & 2015 & 10 & BIL & WW \\
\hline
\end{tabular}


A meta-analysis of QTLs for VW resistance was performed using Biomercator V3 software ([62] (http:// moulon.inra.fr/index.php/fr/equipestransversales/atelier-debioinformatique/projects/ projets/135). Briefly, using Biomercator V3, the map file and QTL file from each study were loaded into the software in the tab delimited format, and were then mapped to the consensus ["Guazuncho2" (G. hirsutum) × "VH8-4602" (G. barbadense)] map [63] obtained from the Cotton Marker Database [64]. Since the map file contains distances between markers on each chromosome, each population's QTLs were mapped to the consensus map separately. A detailed description in the meta-analysis of resistance QTLs can be found in Said et al. $[65,66]$.

To reduce errors in declaring a QTL, four or more QTLs (with a false positive rate of $6.25 \%$ or below) in an interval of $25 \mathrm{cM}$ were considered a consistent QTL region. If there was more than one trait involved in the QTLs, the region is called a QTL cluster. Otherwise, it is called a QTL hotspot for the region involving only one single trait.

\section{Correlation analysis between number of QTLs and nucleotide-binding site (NBS)-encoding genes distributed on chromosomes}

Based on the recent completion of genome sequencing of G. raimondii and G. arboreum [24-26], NBSencoding genes were identified. For example, chromosomes 1 through 13 of the G. raimondii genome carried $24,22,11,8,5,5,87,32,23,5,34,1$, and 8 , respectively [67]. A simple correlation analysis was performed between the number of the NBS genes and the number of total QTLs and QTLs resistant to individual diseases. Coefficients of correlation were tested for significance at the degree of freedom of 24 (genomewide with 26 chromosomes) or 11 (on the subgenome level with 13 chromosomes).

\section{Conclusions}

In this study, linkage mapping of Verticillium wilt (VW) resistance and meta-analysis of QTLs were used to map QTL clusters and hotspots for resistance to VW, Fusarium wilt, root-knot nematodes and reniform nematodes in cotton. In a four-year replicated test of a backcross inbred line population for VW resistance, 10 resistance QTLs were mapped based on a 2895 cM linkage map with 392 SSR markers, which has added useful information to the understanding of the genetic basis of VW resistance in cotton. Twenty-eight disease resistance QTL clusters and 24 hotspots identified from a total of 306 reported QTLs in 32 papers and linked SSR markers provide important information for marker-assisted selection and high resolution mapping of resistance QTLs and genes. The non-overlapping of most resistance QTL hotspots for different diseases indicates that their resistances are controlled by different genes.

\section{Additional file}

Additional file 1: Mapping of quantitative trait loci for Verticillium wilt resistance in a backcross inbred line population of (SG $747 \times$ Giza 75) $\times$ SG $747 \mathrm{BC}_{2} \mathrm{~F}_{4}$.

\section{Abbreviations}

BB: Bacterial blight; BIL: Backcross inbred line; BRR: Black root rot; FW: Fusarium wilt; NBS: Nucleotide binding site; QTL: Quantitative trait locus (QTL); RKN: Root-knot nematodes; RN: Reniform nematodes; SNP: Single nucleotide polymorphism; SSR: Simple sequence repeat; $\mathrm{WW}$ : Verticillium wilt.

\section{Competing interests}

The authors declare that they have no competing interests.

\section{Authors' contributions}

JFZ and JWY conceived the study. JWY, WFP and XLL performed the field experiments. JIS and MZS performed the meta-analysis of QTLS. JFZ and SS wrote the manuscripts. All authors read and approved the manuscripts.

\section{Acknowledgements}

The project was funded in part by the USDA-ARS, Cotton Incorporated and the New Mexico Agricultural Experiment Station.

\section{Author details}

${ }^{1}$ Department of Plant and Environmental Sciences, New Mexico State University, Las Cruces, NM 88003, USA. ${ }^{2}$ State Key Laboratory of Cotton Biology, Institute of Cotton Research of China, Chinese Academy of Agricultural Science, Anyang, Henan 455000, China. ${ }^{3}$ Department of Computer Science, New Mexico State University, Las Cruces, NM 88003, USA. ${ }^{4}$ Department of Entomology, Plant Pathology and Weed Science, New Mexico State University, Las Cruces, NM 88003, USA.

Received: 4 February 2015 Accepted: 1 June 2015

Published online: 05 August 2015

\section{References}

1. Hillocks RJ. Cotton diseases. Wallingford, UK: CAB International; 1992.

2. Kirkpatrick TL, Rothrock CS. Compendium of cotton diseases. St. Paul, MN: Amer. Phytopath. Soc; 2001.

3. Blasingame D, Patel MV. Cotton disease loss estimate committee report. San Antonio, TX: Proc, Beltwide Cotton Conf; 2013. p. 1242-5.

4. Zhang JF, Fang H, Zhou HP, Sanogo S, Ma ZY. Genetics, breeding, and marker-assisted selection for Verticillium wilt resistance in cotton. Crop Sci. 2014;54:1289-303

5. Sanogo S, Zhang JF. Resistance sources, resistance screening techniques and disease management for Fusarium wilt in cotton. Euphytica. 2015 (Accepted)

6. Wilhelm S, Sagen JE, Tietz H. Resistance to Verticillium wilt in cotton: sources, techniques of identification, inheritance trends, and the resistance potential of multilinecultivars. Phytopathology. 1974;64:924-31.

7. Zhang JF, Sanogo S, Flynn R, Baral JB, Bajaj S, Hughs SE, et al. Germplasm evaluation and transfer of Verticillium wilt resistance from Pima (Gossypium barbadense) to Upland cotton (G. hirsutum). Euphytica. 2012;187:147-60.

8. Zhou HP, Fang H, Sanogo S, Hughs SE, Jones DC, Zhang JF. Evaluation of Verticillium wilt resistance in commercial cultivars and advanced breeding lines of cotton. Euphytica. 2014;196:437-48.

9. Zhang JF, Percy RG, MCCarty Jr JC. Introgression genetics and breeding between Upland and Pima cotton- a review. Euphytica. 2014;198:1-12.

10. Zhang JF, Sanogo S, Ma ZY, Qu YY. Breeding, genetics, and quantitative trait locus mapping for Fusarium wilt resistance. Crop Sci doi:10.2135/ cropsci2015.01.0056.

11. Shepherd RL. Transgressive segregation for root-knot nematode resistance in cotton. Crop Sci. 1974;14:872-5. 
12. McPherson MG, Jenkins JN, Watson CE, McCarty Jr JC. Inheritance of root-knot nematode resistance in M-315 RNR and M78-RNR cotton. J Cotton Sci. 2004;8:154-61.

13. Gutiérrez OA, Jenkins JN, McCarty JC, Wubben MJ, Hayes RW, Callahan FE. SSR markers closely associated with genes for resistance to root-knot nematode on chromosomes 11 and 14 of Upland cotton. Theor Appl Genet. 2010;121:1323-37.

14. He Y, Kumar P, Shen X, Davis RF, Van Becelaere G, May OL, et al. Re-evaluation of the inheritance for root-knot nematode resistance in the Upland cotton germplasm line M-120 RNR revealed two epistatic QTLs conferring resistance. Theor Appl Genet. 2014;127:1343-51.

15. Wang C, Ulloa M, Mullens TR, Yu JZ, Roberts PA. QTL Analysis for transgressive resistance to root-knot nematode in interspecific cotton (Gossypium spp.) progeny derived from susceptible parents. PLoS One. 2012;7:e34874

16. Robinson AF, Bell AA, Dighe ND, Menz MA, Nichols RL, Stelly DM. Introgression of resistance to nematode Rotylenchulus reniformis into upland cotton (Gossypium hirsutum) from Gossypium longicalyx. Crop Sci. 2007:47:1865-77.

17. Dighe ND, Robinson AF, Bell AA, Menz MA, Cantrell RG, Stelly DM. Linkage mapping of resistance to reniform nematode in cotton following introgression from Gossypium longicalyx (Hutch. \& Lee). Crop Sci. 2009:41:1151-64.

18. Robinson AF, Bridges AC, Percival AE. New sources of resistance to the reniform (Rotylenchulus reniformis) and root-knot (Meloidogyne incognita) nematode in upland (Gossypium hirsutum) and sea island ( $G$. barbadense) cotton. J Cotton Sci. 2004:8:191-7.

19. Gutiérrez OA, Robinson AF, Jenkins JN, McCarty JC, Wubben MJ, Callahan FE, et al. Identification of QTL regions and SSR markers associated with resistance to reniform nematode in Gossypium barbadense L. accession GB713. Theor Appl Genet. 2011;122:271-80.

20. Buyyarapu R, McPherson M, Parliament K, Kumpatla S, Channabasavaradhya C, Anderson D, et al. Marker assisted selection for reniform nematode resistance using SNP markers in cotton, ICGI meeting. 2014

21. Romano GB, Sacks EJ, Stetina SR, Robinson AF, Fang DD, Gutierrez OA, et al. Identification and genomic location of a reniform nematode (Rotylenchulus reniformis) resistance locus (Ren ${ }^{\text {ari }}$ ) introgressed from Gossypium aridum into upland cotton (G. hirsutum). Theor Appl Genet. 2009;120:139-50

22. Meyers BC, Kaushik S, Nandety RS. Evolving disease resistance genes. Curr Opin Plant Biol. 2005;8:129-34.

23. Sanseverino W, Hermoso A, D'Alessandro R, Vlasova A, Andolfo G, Frusciante L, et al. PRGdb 2.0: towards a community-based database model for the analysis of R-genes in plants. Nucl Acids Res. 2013;41(Database issue):D1167-1171

24. Li F, Fan G, Wang K, Sun F, Yuan Y, Song G, et al. Genome sequence of the cultivated cotton Gossypium arboreum. Nat Genet. 2014;46:567-72.

25. Paterson AH, Wendel JF, Gundlach $H$, Guo H, Jenkins J, Jin D, et al. Repeated polyploidization of Gossypium genomes and the evolution of spinnable cotton fibres. Nature. 2012:492:423-7.

26. Wang K, Wang Z, Li F, Ye W, Wang J, Song G, et al. The draft genome of a diploid cotton Gossypium raimondii. Nat Genet. 2012:44:1098-103.

27. Yu J, Zhang K, Li S, Yu S, Zhai H, Wu M, et al. Mapping quantitative trait loci for lint yield and fiber quality across environments in a Gossypium hirsutum $\times$ Gossypium barbadense backcross inbred line population. Theor Appl Genet. 2013;126:275-87.

28. Wright RJ, Thaxton PM, El-Zik KM, Paterson AH. D-subgenome bias of Xcm resistance genes in tetraploid Gossypium (Cotton) suggests that polyploid formation has created novel avenues for evolution. Genetics. 1998;149:1987-96.

29. Fang $H$, Zhou H, Sanogo S, Flynn R, Percy RG, Hughs SE, et al. Quantitative trait locus mapping for Verticillium wilt resistance in a backcross inbred line population of cotton (Gossypium hirsutum $\times$ Gossypium barbadense). Euphytica. 2013;194:79-91.

30. Fang $H$, Zhou $H$, Sanogo $S$, Lipka AE, Fang DD, Percy RG, et al. Quantitative trait locus analysis of Verticillium wilt resistance in an introgressed recombinant inbred line population of Upland cotton. Mol Breed. 2014;33:709-20.

31. Hinchliffe D, Lu YZ, Potenza C, Gopalan CS, Cantrell RG, Zhang JF. Resistance gene analogues (RGAs) are mapped on homoeologous chromosomes in cultivated tetraploid cotton. Theor Appl Genet. 2005:110:1074-85.
32. Niu C, Lu YZ, Yuan YL, Percy RG, Ulloa M, Zhang JF. Mapping resistance gene analogs (RGAs) in cultivated tetraploid cotton using RGA-AFLP analysis. Euphytica. 2008;181:65-76.

33. Ren G, Li X, Lin Z. Mining, genetic mapping and expression analysis of EST-derived resistance gene homologs (RGHs) in cotton. BMC Plant Biol. 2014;14:203.

34. Percy RG, Kohel RJ. qualitative genetics. In: Smith CW, Cothren JT, editors. Cotton: Origin, History, Technology and Production. John Wiley \& Sons, Inc; 1999. p. p339.

35. St Clair DA. Quantitative disease resistance and quantitative resistance Loci in breeding. Annu Rev Phytopathol. 2010;48:247-68.

36. Benson JM, Poland JA, Benson BM, Stromberg EL, Nelson RJ. Resistance to gray leaf spot of maize: genetic architecture and mechanisms elucidated through nested association mapping and near-isogenic line analysis. PLoS Genet. 2015;11:e1005045.

37. Zhang JF, Stewart JMD. Economic and rapid method for extracting cotton genomic DNA. J Cotton Sci. 2000:4:193-201.

38. Stam P. Construction of integrated genetic linkage maps by means of a new computer package: JoinMap. Plant J. 1993;3:739-44.

39. Li H, Ye G, Wang J. A modified algorithm for the improvement of composite interval mapping. Genetics. 2007;175:361-74.

40. McCouch SR, Cho YG, Yano PE, Blinstrub M, Morishima H, Kinoshita T. Report on QTL nomenclature. Rice Genet Newslett. 1997;14:11-3.

41. Bolek Y, El-Zik KM, Pepper AE, Bell AA, Magill CW, Thaxton PM, et al. Mapping of Verticillium wilt resistance genes in cotton. Plant Sci. 2005;168:1581-90

42. Ge HY, Wang YC, Guo WZ, Zhang TZ. Inheritance and molecular tagging of resistance against Verticillium wilt in Upland cotton. Cotton Sci. 2008;20:19-22.

43. Jiang F, Zhao J, Zhou L, Guo W, Zhang T. Molecular mapping of Verticillium wilt resistance QTL clustered on chromosomes D7 and D9 in Upland cotton. Sci China C Life Sci. 2009;52:872-84.

44. Ning ZY, Zhao R, Chen H, Ai NJ, Zhang X, Zhao J, et al. Molecular tagging of a major quantitative trait locus for broad-spectrum resistance to Verticillium wilt in Upland cotton cultivar Prema. Crop Sci. 2013:53:2304-12.

45. Wang FR, Liu RZ, Wang LM, Zhang CY, Luo GD, Liu QH, et al. Molecular markers of Verticillium wilt resistance in Upland (Gossypium hirsutum L.) cotton cultivar and their effects on assisted phenotypic selection. Cotton Sci. 2007;19:424-30.

46. Wang HM, Lin ZX, Zhang XL, Chen W, Guo XP, Nie YC, et al. Mapping and quantitative trait loci analysis of Verticillium wilt resistance genes in cotton. J Integr Plant Biol. 2008;50:174-82.

47. Wang P, Ning Z, Lin L, Chen H, Mei H, Zhao J, et al. Genetic dissection of tetraploid cotton resistant to Verticillium wilt using interspecific chromosome segment introgression lines. The Crop J. 2014;2:278-88.

48. Yang C, Guo WZ, Li GY, Gao F, Lin SS, Zhang TZ. QTLs mapping for Verticillium wilt resistance at seedling and maturity stages in Gossypium barbadense L. Plant Sci. 2008;174:290-8.

49. Zhang X, Yuan Y, Wei Z, Guo X, Guo Y, Zhang S, et al. Molecular mapping and validation of a major QTL conferring resistance to a defoliating isolate of verticillium wilt in cotton (Gossypium hirsutum L.). PLoS One. 2014;9:e96226.

50. Zhao Y, Wang H, Chen W, Li Y. Genetic structure, linkage disequilibrium and association mapping of Verticillium wilt resistance in elite cotton (Gossypium hirsutum L.) germplasm population. PLoS One. 2014;9:e86308.

51. Lopez-Lavalle LAB, Gillespie VJ, Tate WA, Ellis MH, Stiller WN, Llewellyn, et al. Molecular mapping of a new source of Fusarium wilt resistance in tetraploid cotton (Gossypium hirsutum L.). Mol Breed. 2012;30:1181-91.

52. Mei HX, Ai NJ, Zhang X, Ning ZY, Zhang TZ. QTLs conferring FOV 7 resistance detected by linkage and association mapping in Upland cotton. Euphytica. 2014;197:237-49.

53. Ulloa M, Hutmacher RB, Roberts PA, Wright SD, Nichols RL, Michael Davis R Inheritance and QTL mapping of Fusarium wilt race 4 resistance in cotton. Theor Appl Genet. 2013;126:1405-18.

54. Ulloa M, Wang C, Hutmacher RB, Wright SD, Davis RM, Saski CA, et al. Mapping Fusarium wilt race 1 resistance genes in cotton by inheritance, QTL and sequencing composition. Mol Genet Genomics. 2011;286:21-36.

55. Wang C, Roberts PA. A Fusarium wilt resistance gene in Gossypium barbadense and its effect on root-knot nematode-wilt disease complex. Phytopathology. 2006;96:727-34.

56. Wang P, Su L, Qin L, Hu B, Guo W, Zhang T. Identification and molecular mapping of a Fusarium wilt resistant gene in upland cotton. Theor Appl Genet. 2009;119:733-9. 
57. Shen X, Van Becelaere G, Kumar P, Davis RF, May OL, Chee P. QTL mapping for resistance to root-knot nematodes in the M-120 RNR Upland cotton line (Gossypium hirsutum L.) of the Auburn 623 RNR source. Theor Appl Genet. 2006;11:1539-49.

58. Shen X, He Y, Lubbers EL, Davis RF, Nichols RL, Chee PW. Fine mapping QMi-C11 a major QTL controlling root-knot nematodes resistance in Upland cotton. Theor Appl Genet. 2010;121:1623-31.

59. Ulloa M, Wang C, Roberts PA. Gene action analysis by inheritance and quantitative trait loci mapping of resistance to root-knot nematodes in cotton. Plant Breed. 2009;129:541-50.

60. Wang C, Ulloa M, Roberts PA. Identification and mapping of microsatellite markers linked to a root-knot nematode resistance gene ( $r k n 1)$ in Acala NemX cotton (Gossypium hirsutum L.). Theor Appl Genet. 2006;112:770-6.

61. Niu C, Lister HE, Nguyen B, Wheeler TA, Wright RJ. Resistance to Thielaviopsis basicola in the cultivated A genome cotton. Theor Appl Genet. 2008;117:1313-23.

62. Arcade A, Labourdette A, Falque M, Mangin B, Chardon F, Charcosset A, et al. BioMercator: integrating genetic maps and QTL towards discovery of candidate genes. Bioinformatics. 2004;20:2324-6.

63. Lacape JM, Jacobs J, Arioli T, Derijcker R, Forestier-Chiron N, Llewellyn D, et al. A new interspecific, Gossypium hirsutum $\times$ G. barbadense, RIL population: towards a unified consensus linkage map of tetraploid cotton. Theor Appl Genet. 2009;119:281-92.

64. Blenda A, Scheffler J, Sheffler B, Palmer M, Lacape JM, Yu JZ, et al. CMD: a cotton microsatellite database resource for Gossypium genomics. BMC Genomics. 2006;7:132

65. Said JI, Lin ZX, Zhang XL, Song MZ, Zhang JF. Comprehensive meta QTL analysis for fiber quality, yield, yield related and morphological traits, drought tolerance, and disease resistance in tetraploid cotton. BMC Genomics. 2013;14:776.

66. Said Jl, Song MZ, Wang HT, Lin ZX, Zhang XL, Fang DD, Zhang JF: A comparative meta-analysis of QTL between intraspecific Gossypium hirsutum and interspecific G. hirsutum $\times$ G. barbadense populations. Mol Genet Genomics 2014 (Published online doi:10.1007/s00438-014-0963-9)

67. Wei HL, Li W, Sun XW, Zhu SJ, Zhu J. Systematic analysis and comparison of nucleotide-binding site disease resistance genes in a diploid cotton Gossypium raimondii. PLoS One. 2013;8:e68435.

\section{Submit your next manuscript to BioMed Central and take full advantage of:}

- Convenient online submission

- Thorough peer review

- No space constraints or color figure charges

- Immediate publication on acceptance

- Inclusion in PubMed, CAS, Scopus and Google Scholar

- Research which is freely available for redistribution 